\title{
$S B$-LABELINGS AND POSETS WITH EACH INTERVAL HOMOTOPY EQUIVALENT TO A SPHERE OR A BALL
}

\author{
PATRICIA HERSH AND KAROLA MÉSZÁROS
}

\begin{abstract}
We introduce a new class of edge labelings for locally finite lattices which we call $S B$-labelings. We prove for finite lattices which admit an $S B$-labeling that each open interval has the homotopy type of a ball or of a sphere of some dimension. Natural examples include the weak order, the Tamari lattice, and the finite distributive lattices.
\end{abstract}

Keywords: poset topology, Möbius function, crosscut complex, Tamari lattice, weak order

\section{INTRODUCTION}

Anders Björner and Curtis Greene have raised the following question (personal communication of Björner; see also [15] by Greene).

Question 1.1. Why are there so many posets with the property that every interval has Möbius function equaling 0,1 or -1 ? Is there a unifying explanation?

This paper introduces a new type of edge labeling that a finite lattice may have which we dub an $S B$-labeling. We prove for finite lattices admitting such a labeling that each open interval has order complex that is contractible or is homotopy equivalent to a sphere of some dimension. This immediately yields that the Möbius function only takes the values $0, \pm 1$ on all intervals of the lattice. The construction and verification of validity of such labelings seems quite readily achievable on a variety of examples of interest. The name $S B$-labeling was chosen with $S$ and $B$ reflecting the possibility of spheres and balls, respectively. This method will easily yield that each interval in the weak Bruhat order of a finite Coxeter group, in the Tamari lattice, and in any finite distributive lattice is homotopy equivalent to a ball or a sphere of some dimension. In particular, this method may be applied to non-shellable examples, as the weak Bruhat order for finite Coxeter groups will demonstrate. Example 5.8 will show that not all finite lattices with Möbius function taking only values $0, \pm 1$ admit an $S B$-labeling. However, $S B$-labeling seems to be a convenient method for studying homotopy type by capturing algebraic structure amongst cover relations and may be useful for further applications to lattices which are endowed with such structure.

A motivation for the notion of an $S B$-labeling came from crystal graphs, an important tool for studying the representation theory of Kac-Moody algebras. The crystal graphs

2010 Mathematics Subject Classification. 05E45, 06A07.

The authors thank the Banff International Research Station for providing a stimulating environment in which they began to collaborate, and specifically acknowledge support from NSF conference grant DMS1101740. The first author was partially supported by NSF grants DMS-1002636, DMS-1200730 and DMS1500987, and the second author was partially supported by an NSF Postdoctoral Research Fellowship, DMS1103933, and NSF grant DMS-1501059. 
coming from highest weight representations of Lie algebras in finite type (and more generally in symmetrizable Kac-Moody type) are in fact Hasse diagrams of partially ordered sets. They are naturally endowed with an edge labeling that by definition meets nearly all of the requirements to be an $S B$-labeling (using the upcoming formulation of an $S B$-labeling known as the index 2 formulation). The notion of an $S B$-labeling has been used in [16] to prove that any pair of elements $u, v$ satisfying $u<v$ with $\mu(u, v) \notin\{0,1,-1\}$ in a finite crystal poset given by a highest weight representation in the simply laced case also has the property that the poset interval $[u, v]$ contains within it a relation amongst the so-called crystal operators that is not implied by Stembridge's local relations from [28]. This allowed Hersh and Lenart in [16]. to discover arbitrarily high degree relations amongst crystal operators that are not implied by lower degree relations through a computer search for intervals with Möbius function taking values other than 0,1 , and -1 .

It would be interesting to know of additional examples of finite (or locally finite) lattices with $S B$-labelings. We have not made a comprehensive search for such examples, but rather have chosen to focus in this paper on some well-known families of lattices with the appropriate Möbius function that seemed to us to be especially interesting classes of posets. See [21] by Henri Mühle for further examples of posets with $S B$-labelings e.g. for posets derived from sortable elements in Coxeter groups.

Section 2 quickly reviews background that will be needed later in the paper. Section 3 gives two different formulations for the definition of an $S B$-labeling, and it is shown here that the first of these two versions of the definition for an $S B$-labeling implies each open interval $(u, w)$ in a finite lattice $L$ is homotopy equivalent to a ball or a sphere, with the homotopy type being that of a sphere if and only if $w$ is a join of atoms of the interval. Section 4 proves that these two formulations of the definition for an $S B$-labeling are equivalent to each other. The value in this comes from the fact that the second formulation is a local condition that appears to be more easily verifiable for families of lattices of interest. Section 5 gives applications: it provides $S B$-labelings for the finite distributive lattices, the weak order of any finite Coxeter group, and the Tamari lattice.

\section{BACKGROUND}

A partially ordered set (poset) $P$ is a lattice if each pair of elements $x, y \in P$ has a unique least upper bound, which we denote $x \vee y$, and a unique greatest lower bound, which we denote $x \wedge y$. We denote by $\hat{0}$ (resp. $\hat{1}$ ) the unique minimal (resp. maximal) element of a finite lattice. A cover relation $u \prec v$ in a poset $P$ is a pair of elements $u<v$ with the further requirement that $u \leq z \leq v$ implies either $u=z$ or $z=v$. A chain $u_{1}<u_{2}<\cdots<u_{k}$ is said to be a saturated chain from $u_{1}$ to $u_{k}$ if each $u_{i}<u_{i+1}$ is a cover relation; a saturated chain is a maximal chain of the poset if additionally there do not exist any elements $x$ satisfying either of the conditions $x<u_{1}$ or $u_{k}<x$, i.e., if the chain is not properly contained in any other chains. A maximal chain $u_{1}<\cdots<u_{k}$ need not be the longest chain from $u_{1}$ to $u_{k}$ in terms of number of elements in the chain, and for non-graded lattices such as the Tamari lattice not all maximal chains are of maximal length. An open interval in $P$, denoted $(u, v)$, is the subposet of elements $z \in P$ satisfying $u<z<v$. Likewise, a closed interval $[u, v]$ is the subposet comprised of those $z \in P$ such that $u \leq z \leq v$. We will sometimes refer to the open interval $(\hat{0}, \hat{1})$ in a finite lattice $L$ as the proper part of $L$. For convenience, 
we pass freely back in forth between speaking of the saturated chains from $u$ to $v$, and the maximal chains of the closed interval $[u, v]$.

The Möbius function, denoted $\mu_{P}$, of a finite partially ordered set $P$ is defined recursively as follows. For each $u \in P$ we have $\mu_{P}(u, u)=1$. For each $u<v, \mu_{P}(u, v)=$ $-\sum_{u \leq x<v} \mu_{P}(u, x)$. The Möbius function provides the coefficients in inclusion-exclusion counting formulas. The order complex of a finite poset $P$ is the simplicial complex, denoted $\Delta(P)$, whose $i$-faces are chains $v_{0}<\cdots<v_{i}$ of $i+1$ comparable poset elements. It is well known for each $u<v$ in $P$ that $\mu_{P}(u, v)=\tilde{\chi}(\Delta(u, v))$ where $\Delta(u, v)$ denotes the order complex of the open interval $(u, v)$ and $\tilde{\chi}$ denotes its reduced Euler characteristic (which is obtained from the usual Euler characteristic by subtracting one from it). Sometimes we will speak of the homotopy type of a poset or poset interval, by which we mean the homotopy type of the order complex of that poset or poset interval.

Our focus throughout this paper will be on posets in which the order complex of each open interval $(u, v)$ will turn out to be homotopy equivalent to a ball or a sphere, implying that $\tilde{\chi}(\Delta(u, v))$ and hence $\mu_{P}(u, v)$ equals 0,1 , or -1 for each pair $u<v$. A key tool underlying our work will be the Crosscut Theorem, which we review next.

Recall from [3] (see also [2], [14], 24]) that a subset $C$ of a poset $P$ is called a crosscut if the following conditions hold.

(1) $C$ is an antichain.

(2) For every finite chain $\sigma$ in $P$ there exists an element of $C$ that is comparable to every element of $\sigma$.

(3) For each $A \subseteq C$ which is bounded, i.e., which has an upper bound or a lower bound, then the join or the meet of the elements of $A$ exists as an element of $P$.

Define the crosscut complex given by a crosscut $C$ to be the simplicial complex whose faces are those subsets of $C$ which are bounded.

Remark 2.1. In a finite lattice $L$ (and hence also in the proper part of $L$ ), the set of atoms of $L$ is a crosscut. In this paper, we will make use of the next theorem with the atoms as the chosen crosscut.

Theorem 2.2 (Crosscut Theorem, Theorem 10.8 in [3]). The crosscut complex given by any crosscut of a finite poset $P$ is homotopy equivalent to the order complex of $P$.

Example 2.3. Letting $B_{n}$ denote the poset of subsets of $\{1,2, \ldots, n\}$ ordered by containment, notice that the crosscut complex for $B_{3}$ given by the crosscut comprised of the atoms of $B_{3}$ is a 2-simplex. On the other hand, consider the subposet $B_{3} \backslash\{\hat{0}, \hat{1}\}$ in which we delete the unique minimal and maximal elements, namely we delete $\hat{0}=\emptyset$ and $\hat{1}=\{1,2,3\}$; this smaller poset has the same crosscut, but its crosscut complex is the boundary of a 2 -simplex.

Remark 2.4. If one can prove that distinct sets of atoms have distinct joins, then the Crosscut Theorem will imply that the subposet of joins of atoms has order complex that is homotopy equivalent to the order complex for the entire poset. We will use this in the special case where our poset is the proper part of a finite lattice. An $S B$-labeling, a new type of edge labeling which we introduce momentarily, will guarantee that distinct sets of atoms have distinct joins. 


\section{A NEW CLASS OF EDGE LABELINGS: $S B$-LABELINGS}

Next we introduce a new class of edge labelings which we call $S B$-labelings. We will call a lattice admitting such a labeling an $S B$-lattice. We will give two different formulations of the definition of an $S B$-labeling, and then we will prove that these are equivalent to each other. One formulation will be convenient for proving topological consequences of having an $S B$-labeling. In particular, we use this formulation to prove that each open interval in a finite lattice with an $S B$-labeling is homotopy equivalent to a ball or a sphere. The other formulation seems likely to be more convenient for constructing $S B$-labelings on examples.

Later in the paper we will indeed demonstrate that several well-known lattices admit $S B$ labelings, in spite of the fact that some of these lattices cannot possibly be shellable. Specifically, we will apply this method of $S B$-labeling to the weak Bruhat order of a finite Coxeter group, to the Tamari lattice, and to the finite distributive lattices, while Example 5.8 will show that dominance order on integer partitions does not in general admit an $S B$-labeling. The $S B$-labelings will yield the homotopy type of each poset interval by a short, uniform approach for these classes of posets which had previously been analyzed by other methods.

Remark 3.1. It is natural to ask if this notion for edge labelings may be extended to a more general notion for chain labelings (in the sense of [7]). However, key properties of these $S B$-labelings in fact will rely in an essential way on our usage of edge labelings rather than chain labelings. Therefore, we confine ourselves to considering edge labelings.

Definition 3.2. An edge labeling $\lambda$ of a finite lattice $L$ is a lower $S B$-labeling if it may be constructed as follows. Begin with a label set $S$ such that there is a subset $\left\{\lambda_{a} \mid a \in A(L)\right\}$ of $S$ whose members are in bijection with the set $A(L)$ of atoms of $L$.

(1) No two labels upward from $\hat{0}$ to distinct atoms may be equal. This allows us to define the label $\lambda_{a}$ on each cover relation $\hat{0} \prec a$ as the label corresponding to the atom $a$.

(2) Given any interval of the form $\left[\hat{0}, a_{i_{1}} \vee \cdots \vee a_{i_{r}}\right]$ for $\left\{a_{i_{1}}, \ldots, a_{i_{r}}\right\} \subseteq A(L)$, each of the saturated chains $M$ from $\hat{0}$ to $a_{i_{1}} \vee \cdots \vee a_{i_{r}}$ has the property that the set $\lambda(M)$ of labels occurring with positive multiplicity on $M$ is exactly $\left\{\lambda_{a_{i_{j}}} \mid 1 \leq j \leq r\right\}$.

When an edge labeling $\lambda$ for a finite lattice $L$ meets these conditions upon restriction to each closed interval of $L$, then we call such a labeling an $S B$-labeling. We call a lattice with an $S B$-labeling an $S B$-lattice.

Remark 3.3. Notice that condition (2) above implies for $S, T$ distinct sets of atoms, that the join of the set of atoms in $S$ does not equal the join of the set of atoms in $T$. In particular, this implies that the subposet of joins of atoms is a Boolean algebra.

Now we give what we call the "index 2 formulation of an $S B$-labeling", a type of labeling that we will prove in Theorem 3.5 is equivalent to the notion of $S B$-labeling. In light of Theorem 3.5, one may henceforth take either definition as a definition of $S B$-labeling.

Definition 3.4. The index 2 formulation of an $S B$-labeling is an edge labeling on a finite lattice $L$ satisfying the following conditions for each $u, v, w \in L$ such that $v$ and $w$ are distinct elements which each cover $u$ :

(i) $\lambda(u, v) \neq \lambda(u, w)$

(ii) Each saturated chain from $u$ to $v \vee w$ uses both of these labels $\lambda(u, v)$ and $\lambda(u, w)$ a positive number of times. 


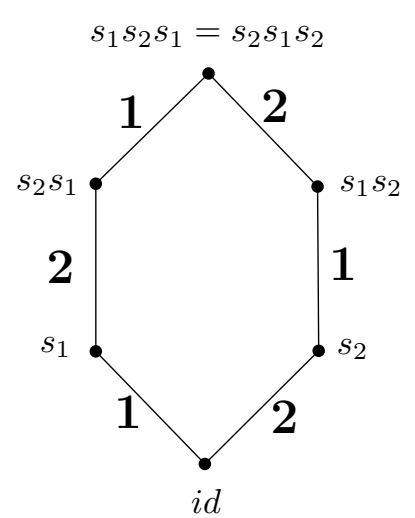

$(a)$

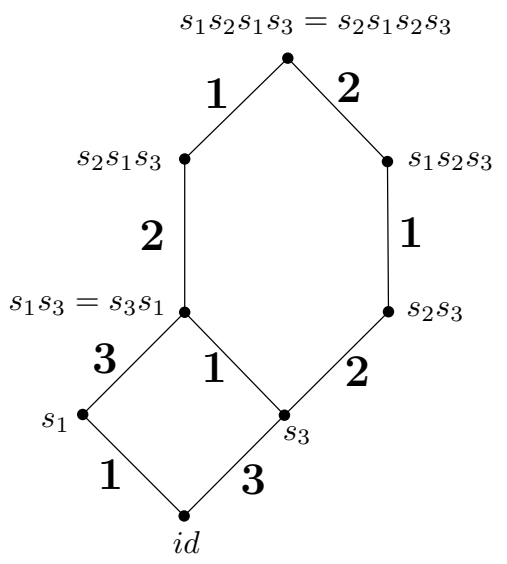

(b)

Figure 1. (a) The $S B$-labeling of the weak Bruhat order of $S_{3}$ as described in Example 3.6. The complex $\Delta\left(i d, s_{1} s_{2} s_{1}\right)$ is homotopy equivalent to a sphere.. (b) The $S B$-labeling of an interval of the weak Bruhat order of $S_{4}$ as described in Example 3.6. The complex $\Delta\left(i d, s_{1} s_{2} s_{1} s_{3}\right)$ is homotopy equivalent to a ball.

(iii) None of the saturated chains from $u$ to $v \vee w$ use any other types of labels besides $\lambda(u, v)$ and $\lambda(u, w)$.

Theorem 3.5. An edge labeling on a finite lattice is an SB-labeling if and only if it satisfies the index 2 formulation for an $S B$-labeling.

Proof. Theorem 4.6 proves that the index 2 formulation of an $S B$-labeling will always give an $S B$-labeling. On the other hand, if $\lambda$ is an $S B$-labeling, then Condition (1) for $S B$-labelings directly gives Condition (i) in the index 2 formulation for an $S B$-labelings. Condition (2) for $S B$-labelings specialized to the case of a join of two atoms yields exactly conditions (ii) and (iii) of the index 2 formulation of an $S B$-labeling.

Example 3.6. In the case of the weak Bruhat order of a finite Coxeter group, we will label each cover relation $u \prec s_{i} u$ with the label $s_{i}$ and will prove that this labeling meets the requirements of the index 2 formulation of an $S B$-labeling. Theorem 5.3 will verify that this is indeed an $S B$-labeling. See Figure 3 for an example. To illustrate part of the subtlety in this definition, notice e.g. that the weak order interval $\left[\hat{0}, s_{1} s_{2}\right]$ has a single maximal chain, and it uses the edge labels $s_{1}$ and $s_{2}$. The label $s_{2}$ corresponds to an atom while the label $s_{1}$ does not. In this weak order interval with only one atom, the conditions above hold vacuously since there is no triple of elements $u, v, w$ as in Definition 3.4 .

Next we show how the property of being an SB-lattice gives topological information about the order complex of each open interval in the lattice.

Theorem 3.7. If $L$ is an SB-lattice, then each open interval is homotopy equivalent to a ball or a sphere of some dimension. Moreover, $\Delta(u, v)$ is homotopy equivalent to a sphere if and only if $v$ is a join of atoms of the interval, in which case it is a sphere $S^{d-2}$ where $d$ is the number of atoms in the interval. 
Proof. First note that it suffices to prove the result for lower intervals $(\hat{0}, v)$, by virtue of how Definition 3.2 is formulated. Now each subset of the atoms has a distinct join, due to the fact that the set of labels appearing on the edges of all of the saturated chains upward from $\hat{0}$ to a join of atoms is exactly that set of atom labels. But this implies (cf. Remark 2.4) that the crosscut complex for $(\hat{0}, v)$ given by the atoms is the boundary of a simplex if $v$ is a join of atoms and is the entire simplex otherwise. In particular, this means that the crosscut complex is homotopy equivalent to a sphere $S^{d-2}$ if $v$ is a join of atoms and is contractible otherwise. Now the Crosscut Theorem (which we recall as Theorem 2.2 yields the result.

We conclude this section with some relaxations that may be made in the hypotheses of our main results without changing the conclusions.

Remark 3.8. In the notion of an $S B$-labeling, we may replace the finiteness requirement for our lattices by instead requiring them to be locally finite with a unique minimal element. Our proofs all go through unchanged in such cases, allowing us to call such lattices $S B$-lattices and draw all of the same conclusions. Young's lattice will provide one such example, as we will show in Section 5 .

Definition 3.9. Let us say that a finite poset $P$ with unique minimal and maximal elements is an atom-near-lattice if each pair of elements $u, v \in P$ with $u<v$ has the property that each collection $S$ of atoms of the closed interval $[u, v]$ has a unique least upper bound $\vee_{a \in S} a$.

Remark 3.10. It is proven in Lemma 2.1 of [6] that this atom-near-lattice property in fact implies that $P$ is a lattice. This property may be easier to check in examples of interest than the property of being a lattice. Our proofs actually only rely upon this formulation of the lattice property.

\section{INDEX 2 FORMULATION IS EQUIVALENT TO AN $S B$-LABELING}

This section proves the equivalence of our two different definitions for an $S B$-labeling. To this end, we will use the next two notions to prove that every labeling meeting the conditions in the index 2 formulation for an $S B$-labeling is an $S B$-labeling.

Definition 4.1. We say that a pair of maximal chains $M_{1}, N_{1}$ in a finite lattice are connected by a basic move if $M_{1}$ and $N_{1}$ coincide except on an open interval $(u, v)$ where $u \prec x$ in $M_{1}$ and $u \prec y$ in $N_{1}$ with $x \neq y$ and with $v=x \vee y$.

Example 4.2. For example, in any interval $[u, w u]$ in the weak order the basic moves are given by the long and short braid moves on reduced expressions for the Coxeter group element $w$.

Definition 4.3. Define the total length of a closed interval $[u, v]$ to be the sum of the lengths of all the saturated chains in that interval.

This notion of total length is designed to enable a proof by induction in the next lemma without needing the lattices to be graded and without needing to require the maximal chains of $[a, b]$ to be of maximal length amongst all maximal chains on $[a, b]$.

Lemma 4.4. Any two maximal chains on an interval $[a, b]$ in a finite lattice $L$ are connected by a series of basic moves. 
Proof. Suppose otherwise. Then choose an interval $[u, v]$ where this fails, making the total length of the interval as small as possible among all such examples. Let $M_{1}$ and $N_{1}$ be two maximal chains on $[u, v]$ that are not connected by a series of basic moves. Our minimality assumption on total length ensures for $u \prec u_{1}$ in $M_{1}$ and $u \prec v_{1}$ in $N_{1}$ that we must have $u_{1} \neq v_{1}$. We also may assume $u_{1} \vee v_{1} \neq v$, since otherwise there would be a single basic move connecting $M_{1}$ to $N_{1}$ by definition of basic move.

Our plan in this case is to give a series of steps $M_{1} \rightarrow M_{2} \rightarrow M_{3} \rightarrow N_{1}$ which convert $M_{1}$ to $N_{1}$ and to show that each of these three steps may be achieved through a series of basic moves, hence that their composition may as well. Let $M_{2}$ be a maximal chain on $[u, v]$ which agrees with $M_{1}$ except possibly on $\left(u_{1}, v\right) ; M_{2}$ is chosen to include $u_{1} \vee v_{1}$ (since $v \neq u_{1} \vee v_{1}$, we have $\left.u_{1} \vee v_{1}<v\right)$. Since the interval $\left[u_{1}, v\right]$ has strictly smaller total length than $[u, v]$ and $M_{1}$ agrees with $M_{2}$ except on this interval, we can conclude there is a series of basic moves converting the restriction of $M_{1}$ to $\left[u_{1}, v\right]$ to the restriction of $M_{2}$ to this same interval, which in turn gives basic moves converting $M_{1}$ to $M_{2}$ in $[u, v]$. Now we similarly may convert $M_{2}$ to a maximal chain $M_{3}$ which coincides with $M_{2}$ except on the interval $\left(u, u_{1} \vee v_{1}\right)$ and which has $u_{1}$ replaced by $v_{1}$; this interval also has strictly smaller total length than $[u, v]$, again implying the desired basic moves. Finally, we note that $M_{3}$ only differs from $N_{1}$ on the proper part of the interval $\left[v_{1}, v\right]$, which yet again has strictly smaller total length than $[u, v]$, enabling us to find a series of basic moves converting $M_{3}$ to $N_{1}$, completing the result.

Remark 4.5. Lemma 4.4 may be regarded as an abstraction of the idea that the lattice property for the weak Bruhat order of a finite Coxeter group ensures that any two reduced expressions for the same Coxeter group element are connected by a series of long and short braid moves. This implication in the case of the weak order appears as Theorem 3.3.1 in [5].

Theorem 4.6. If a finite lattice $L$ has an edge labeling that satisfies the index 2 formulation for an $S B$-labeling, then it is an $S B$-labeling.

Proof. Let $\lambda$ be an edge labeling for a finite lattice $L$ which meets the requirements for the index 2 formulation of an $S B$-labeling. We will prove by induction on the number $r$ of atoms that $\lambda$ also meets the requirements to be a lower $S B$-labeling. In fact, this will imply $\lambda$ is an $S B$-labeling, since applying this argument to any closed interval will show we have a lower $S B$-labeling for each closed interval.

The base case with 1 atom is tautologically true. Let us suppose that $\left\{a_{i_{1}}, \ldots, a_{i_{r}}\right\}$ is the set of atoms of $L$. Now consider the interval $L_{r-1}=\left[\hat{0}, a_{i_{1}} \vee \cdots \vee a_{i_{r-1}}\right]$ within $L$. By induction, we may assume that this uses only the labels $\left\{a_{i_{1}}, \ldots, a_{i_{r-1}}\right\}$. We will progressively build from $L_{r-1}$ a larger subposet $L_{r-1,1}$ of $L$ all of whose cover relations are cover relations of $L$ with the further property that it includes an upper bound $m$ for $\left\{a_{i_{1}} \ldots, a_{i_{r}}\right\}$. We will deduce from $a_{i_{1}} \vee \cdots \vee a_{i_{r}} \leq m$ that $\left[\hat{0}, a_{i_{1}} \vee \cdots \vee a_{i_{r}}\right]$ also uses at most the labels $\left\{a_{i_{1}}, \ldots, a_{i_{r}}\right\}$. Finally, we will also show that each saturated chain from $\hat{0}$ to $a_{i_{1}} \vee \cdots \vee a_{i_{r}}$ in fact uses all of these labels.

First we add to $L_{r-1}$ the additional atom $a_{i_{r}}$ as well as all elements belonging to the closed interval $\left[\hat{0}, a_{i_{1}} \vee a_{i_{r}}\right.$ ] to obtain a new poset $L_{r-1}^{(1)}$. By condition (iii) in the index 2 formulation of an $S B$-labeling, this slightly larger poset still only uses the allowed edge labels, since all of the new cover relations are in the interval $\left[0, a_{i_{1}} \vee a_{i_{r}}\right]$ which only uses the labels $a_{i_{1}}$ and $a_{i_{r}}$. Now either $a_{i_{1}} \vee a_{i_{r}} \in L_{r-1}$, in which case we are done constructing $L_{r-1,1}$, or there are 
at least two different maximal elements in $L_{r-1}^{(1)}$. For each maximal element $m_{i}^{(1)} \in L_{r-1}^{(1)}$, let $P_{i}$ be the subposet of elements $x \in L_{r-1}^{(1)}$ satisfying $x \leq m_{i}^{(1)}$. Choose $u^{(1)}$ to be an element that is contained in both $P_{j}$ and $P_{k}$ for some $j \neq k$ such that there are no elements strictly greater than $u^{(1)}$ also having the property of being contained in some $P_{j^{\prime}}$ as well as some $P_{k^{\prime}}$ for $j^{\prime} \neq k^{\prime}$; finiteness of $L_{r-1}^{(1)}$ guarantees the existence of such an element $u^{(1)}$.

Now consider cover relations $u^{(1)} \prec x_{1}^{(1)}$ and $u^{(1)} \prec x_{2}^{(1)}$ in $L_{r-1}^{(1)}$ such that $x_{1}^{(1)} \leq m_{j}^{(1)}$ and $x_{2}^{(1)} \leq m_{k}^{(1)}$ in $L_{r-1}^{(1)}$. Obtain from $L_{r-1}^{(1)}$ a strictly larger poset $L_{r-1}^{(2)}$ by adding all elements and cover relations from the interval $\left[u^{(1)}, x_{1}^{(1)} \vee x_{2}^{(1)}\right]$. Again by condition (iii), this cannot introduce any new labels. Again, we either have a unique maximal element or we have two different maximal elements, allowing us to apply this same procedure and do so repeatedly until we have a unique maximal element $m$. Specifically, at the $n$-th iteration of the procedure, the input is a poset $L_{r-1}^{(n-1)}$ having distinct maximal elements. This allows us to find an element $u^{(n)}$ satisfying the same criterion at this step that $u^{(1)}$ satisfied at the first step, now using distinct maximal elements $m_{j}^{(n-1)}$ and $m_{k}^{(n-1)}$ from $L_{r-1}^{(n-1)}$. This in turn ensures there are elements $x_{1}^{(n)}$ and $x_{2}^{(n)}$ with $u^{(n)} \prec x_{1}^{(n)} \leq m_{j}^{(n-1)}$ and $u^{(n)} \prec x_{2}^{(n)} \leq m_{k}^{(n-1)}$ such that $x_{1}^{(n)}$ is not less than or equal to any maximal element other than $m_{j}^{(n-1)}$, and $x_{2}^{(n)}$ is not less than or equal to any maximal element other than $m_{k}^{(n-1)}$. We obtain $L_{r-1}^{(n)}$ as the poset $L_{r-1}^{(n-1)}$ with the additional elements and cover relations from the interval $\left[u^{(n)}, x_{1}^{(n)} \vee x_{2}^{(n)}\right]$ added to it.

We iterate this process until it yields a poset $L_{r-1,1}$ with a unique maximal element $m$. This process must terminate within finitely many iterations due to finiteness of our original lattice. By construction, the unique maximal element $m$ of $L_{r-1,1}$ will be an upper bound for $\left\{a_{i_{1}}, \ldots, a_{i_{r}}\right\}$, and we will have only used the labels $a_{i_{1}}, \ldots, a_{i_{r}}$ on the poset $L_{r-1,1}$ obtained by this process. The fact that we only ever insert cover relations from the original lattice implies that each saturated chain in $L_{r-1,1}$ from $\hat{0}$ to $m$ is also a saturated chain in the original lattice $L$. Since $a_{i_{1}} \vee \cdots \vee a_{i_{r}} \leq m$ in $L$, there is a saturated chain from $\hat{0}$ to $m$ in $L$ which includes the element $a_{i_{1}} \vee \cdots \vee a_{i_{r}}$. By Lemma 4.9, this implies that the set of labels on each saturated chain from $\hat{0}$ to $a_{i_{1}} \vee \cdots \vee a_{i_{r}}$ must be a subset of the set of labels on a saturated chain from $\hat{0}$ to $m$. Thus, no labels other than $a_{i_{1}}, \ldots, a_{i_{r}}$ appear on any saturated chain from $\hat{0}$ to $a_{i_{1}} \vee \cdots \vee a_{i_{r}}$.

Now let us show that each saturated chain from $\hat{0}$ to $a_{i_{1}} \vee \cdots \vee a_{i_{r}}$ uses each of the labels $\left\{a_{i_{1}}, \ldots, a_{i_{r}}\right\}$ a positive number of times. The point is that each atom $a_{i_{j}}$ for $1 \leq j \leq r$ is in some maximal chain in $\left[\hat{0}, a_{i_{1}} \vee \cdots \vee a_{i_{r}}\right]$, implying that there exists a maximal chain using the label $a_{i_{j}}$; but this implies that all maximal chains use $a_{i_{j}}$ for each $1 \leq j \leq r$, by Lemma 4.9. In conclusion, we have shown that each maximal chain uses exactly the set of labels $\left\{a_{i_{1}}, \ldots, a_{i_{r}}\right\}$, each with positive multiplicity.

Corollary 4.7. An edge labeling of a finite lattice is an SB-labeling if and only if it satisfies the index 2 formulation of an SB-labeling.

Proof. One direction is proven as Theorem 4.6. The other direction follows immediately from the fact that the index 2 formulation of an $S B$-labeling is a special case of the notion of an $S B$-labeling. 
To complete the proof of Theorem 4.6, what remains is to prove Lemma 4.9, using Lemma 4.8 below to do this.

Lemma 4.8. Let $L$ be a finite lattice with an edge labeling $\lambda$ which satisfies the index 2 formulation for an $S B$-labeling. Then for any $u, v, w \in L$ with $v$ and $w$ both covering $u$, the interval $[u, v \vee w]$ cannot have any atoms other than $v$ and $w$.

Proof. If there were another atom $x$ in $[u, v \vee w]$, then we must have $\lambda(u, x) \neq \lambda(u, v)$ and $\lambda(u, x) \neq \lambda(u, w)$. We also must have $x \vee v \leq v \vee w$ since $x \leq v \vee w$ and $v \leq v \vee w$. By virtue of $\lambda$ meeting the index 2 formulation for an $S B$-labeling, we have that every maximal chain on the interval $[u, x \vee v]$ must use the label $\lambda(u, x)$. But then there will be maximal chains on the interval $[u, v \vee w]$ which also must use the label $\lambda(u, x)$, a contradiction.

Lemma 4.9. If an edge labeling $\lambda$ on a finite lattice $L$ meets the conditions for the index 2 formulation of an $S B$-labeling, then this guarantees for each interval $[a, b]$ in $L$ that any two saturated chains $M_{1}$ and $N_{1}$ on $[a, b]$ must use the same set of labels each a positive number of times, though not necessarily with the same multiplicities.

Proof. Lemma 4.8 implies that any two maximal chains $M_{1}$ and $N_{1}$ on an interval $[a, b]$ in a finite lattice that are connected by a series of basic moves use the same set of labels (though not necessarily with the same multiplicities). Lemma 4.4 checks that any two saturated chains $M_{1}$ and $N_{1}$ from $a$ to $b$ in a finite lattice are connected by a series of basic moves, so the result follows.

\section{Applications}

Now we turn to applications, beginning with finite distributive lattices. In this first example, the $S B$-labeling we give is also a well-known $E L$-labeling, implying the posets are shellable. The homotopy type of the intervals in finite distributive lattices was determined in [1] indirectly by virtue of finite distributive lattices also being finite supersolvable lattices, relying on an earlier $R$-labeling given by Stanley in [25] for finite supersolvable lattices.

Theorem 5.1. Any finite distributive lattice is an SB-lattice.

Proof. We will use the fact that any finite distribute lattice $L$ is the poset $J(P)$ of order ideals of a finite poset $P$ ordered by inclusion (cf. [26], Theorem 3.4.1). This allows us to regard each cover relation $u \prec v$ as adding to the order ideal associated to $u$ a single element $p \in P$ We use this element $p$ as the label for $u \prec v$. Whenever we have $u \prec v$ and $u \prec w$, then this implies that there are two different elements of $P$, either of which may individually be added to the order ideal given by $u$ to obtain a new order ideal. Therefore, $v \vee w$ covers both $v$ and $w$ with the further property that there cannot be any other elements $z$ with $u<z<v \vee w$. From this, Conditions (i), (ii) and (iii) for the index 2 formulation for an $S B$-labeling follow directly.

Recall that Young's lattice is the poset of integer partitions regarded as Young diagrams, with $u \prec v$ whenever $v$ is obtained from $u$ by adding a single box. Since Young's lattice is a locally finite, distributive lattice with a unique minimal element, Theorem 5.1 together with Remark 3.8 allows us to conclude:

Corollary 5.2. Young's lattice is an SB-lattice. 
Next we turn to a non-shellable example, the weak Bruhat order on the elements of a finite Coxeter group $W$. Let $S$ be the set of simple reflections generating $W$. The (left) weak Bruhat order has as its cover relations each $w \prec s_{i} w$ for $w \in W$ and $s_{i} \in S$ with $l(w)<l\left(s_{i} w\right)$, letting $l(w)$ denote the Coxeter-theoretic length of $w$. See e.g. [5] or [18] for further background on Coxeter groups and on the weak Bruhat order. The homotopy type of each interval was originally determined in [4] (see also [11] and [13] for related results regarding posets of regions). We will use the following properties of weak Bruhat order.

(1) There is an isomorphism of weak Bruhat order intervals $[u, w] \simeq\left[e, w u^{-1}\right]$ for each $u \leq w$. This appears as Proposition 3.1.6 in [5].

(2) There is a characterization of the joins of finite sets of atoms in Lemma 3.2 .3 in [5] as exactly those Coxeter group elements which are longest elements of parabolic subgroups of $W$; specifically, the join of the collection of atoms corresponding to the simple reflections in $S=\left\{s_{i_{1}}, \ldots, s_{i_{r}}\right\}$ will be $w_{\circ}(S)$, namely the longest element of the parabolic subgroup $W_{S}$ generated by the elements of $S$.

(3) There is a bijection between the maximal chains in the interval $[u, w]$ and the reduced expressions for $w u^{-1}$. This appears in Proposition 3.1.2, part (i), in [5].

(4) The atoms of $\left[e, w_{\circ}(S)\right]$ are exactly the atoms $a_{s}$ for $s \in S$, namely the atoms naturally corresponding to the elements of $S$. A proof of this may be found in Propositions 3.1.2, parts (iii) and (iv) in [5].

(5) The weak Bruhat order is a lattice. This is proven e.g. in Theorem 3.2.1 in [5]

Theorem 5.3. The weak Bruhat order for any finite Coxeter group $W$ is an SB-lattice. Moreover, an open interval $(u, w)$ in $W$ is homotopy equivalent to a sphere $S^{d-2}$ if $w u^{-1}$ is the longest element of a parabolic subgroup $W_{S}$ with $|S|=d$, and $(u, w)$ is contractible otherwise.

Proof. We will prove the result for left weak Bruhat order, noting that a completely analogous proof holds for right weak Bruhat order. By property (5) above, the weak Bruhat order of a finite Coxeter group is a lattice. To obtain an $S B$-labeling, we will label each cover relation $u \prec v$ with the unique simple reflection $s_{i}$ such that $v=s_{i} u$.

Next let us justify the following claim: for any two distinct cover relations $u \prec v$ and $u \prec w$, there are unique saturated chains $u \prec v \prec \cdots \prec v \vee w$ and $u \prec w \prec \cdots \prec v \vee w$ from $u$ to $v \vee w$ and no other saturated chains from $u$ to $v \vee w$; moreover, these two saturated chains have label sequences $s_{i} s_{j} s_{i} \cdots$ and $s_{j} s_{i} s_{j} \cdots$ each consisting of an alternation of only the letters $s_{i}$ and $s_{j}$, with each label sequence having the same length $m(i, j)$ where $m(i, j)$ is the order of the Coxeter group element $s_{i} s_{j}$. In the case with $u=e$, this claim holds by property (2) above. Otherwise, we use property (1) above to reduce the claim to the case with $u=e$. This claim shows that all of the requirements for the index 2 formulation of an $S B$-labeling are indeed satisfied, implying that weak Bruhat order is an $S B$-lattice.

Properties (1) and (2) above also combine to imply that $w$ will be a join of atoms of the interval $[u, w]$ if and only if $w u^{-1}$ is the longest element $w_{\circ}\left(W_{S}\right)$ of some parabolic subgroup $W_{S}$ of $W$. Letting $A$ be the set of atoms of $[u, w]$, Theorem 3.7 implies that $[u, w]$ has the homotopy type of a sphere $S^{|A|-2}$ if and only if $w u^{-1}$ is the longest element of some parabolic subgroup $W_{S}$ of $W$ and is contractible otherwise. In the case that $w u^{-1}=w_{\circ}\left(W_{S}\right)$, property (4) above guarantees that $|A|=|S|$. 


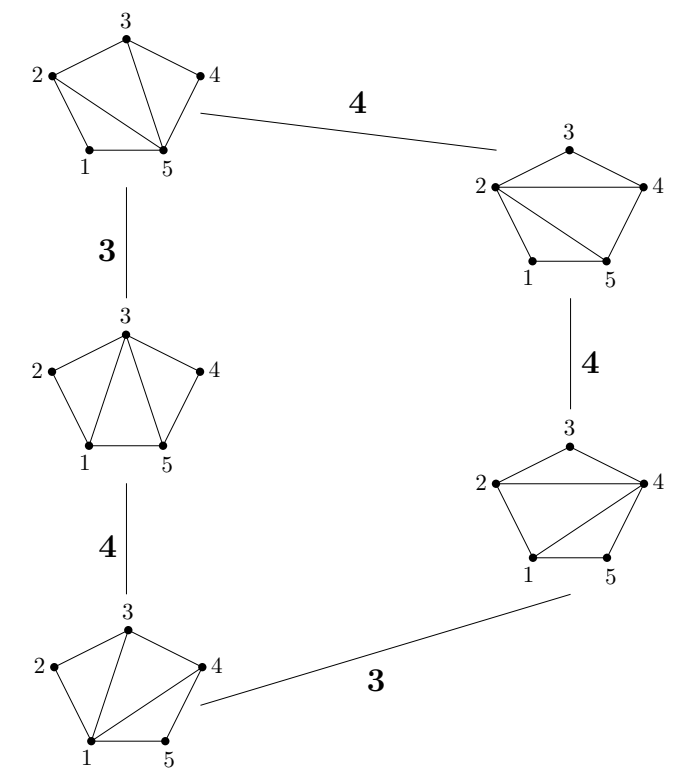

Figure 2. The Tamari lattice for $n=4$ with the $S B$-labeling from the proof of Theorem 5.5 .

The Tamari lattice, our next example, is a partial order on the triangulations of an $(n+1)$ gon, as discussed e.g. in [12]. To define the cover relations, it is convenient to label the $n+1$ vertices of the $(n+1)$-gon with the integers $1,2, \ldots, n+1$, proceeding clockwise about the boundary of the $(n+1)$-gon. Now each cover relation $u \prec v$ will replace an edge $e_{i, k}$ in a triangulation $u$ by an edge $e_{j, l}$ to obtain the triangulation $v$, subject to the requirement that we have $i<j<k<l$. See Figure 2 for an illustration.

The significance of the Tamari lattice comes in part from the fact that its Hasse diagram is the 1-skeleton of the associahedron, a polytope which goes back to work on homotopy associative $H$-spaces by Stasheff (cf. [27]). The number of elements in the Tamari lattice is a Catalan number. It was proven to be non-pure shellable with each interval having the homotopy type of a ball or a sphere by Björner and Wachs in [8]. Earlier results regarding its Möbius function and implicitly regarding its topological structure also appear in [23]. See e.g. [8], [17], 20] for further background on the Tamari lattice.

Remark 5.4. The symmetry in this definition for the Tamari lattice guarantees that it will be self-dual, so that having an $S B$-labeling will be equivalent to its dual poset having an $S B$-labeling.

Theorem 5.5. The Tamari lattice is an SB-lattice.

Proof. For our proposed $S B$-labeling $\lambda$, we record for each cover relation $v \prec w$ the letter $\lambda(v, w)=k$ such that an edge $e_{i, k}$ in $v$ is replaced by an edge $e_{j, l}$ to obtain $w$ for some $i<j<k<l$. Lemma 5.6 proves that there is at most one cover relation upward from $v$ with this label $k$, thereby proving condition (i) in the index 2 formulation for an $S B$-labeling. Let us denote by $u_{k}(v)$ the element $w$ with $v \prec w$ and $\lambda(v, w)=k$, when such an element $w$ exists. 
In order to prove conditions (ii) and (iii) in the index 2 formulation for an $S B$-labeling, it will suffice to confirm the following facts regarding any element $v$ in the Tamari lattice and any pair of distinct elements $u_{i}(v)$ and $u_{j}(v)$ both covering $v$ with $i<j$.

(1) We have either $u_{i} u_{j}(v)=u_{j} u_{i}(v)$ or $u_{i} u_{j}(v)=u_{j} u_{j} u_{i}(v)$; moreover, $u_{i}(v) \vee u_{j}(v)=$ $u_{i} u_{j}(v)$ in either case.

(2) The only possible elements in the interval $\left[v, u_{i}(v) \vee u_{j}(v)\right]$ are $v, u_{i}(v), u_{j}(v), u_{i} u_{j}(v)$ and $u_{j} u_{i}(v)$.

Both of these claims are proven in Lemma 5.7 .

Lemma 5.6. Each element $v$ is covered by at most one element $w$ such that $\lambda(v, w)=k$ for any given label $k$.

Proof. Given a triangulated $(n+1)$-gon $v$, consider the edges emanating outward from some fixed vertex $k$ for $2 \leq k \leq n$, proceeding in counterclockwise order through this list of edges, and including in it the boundary edges $e_{k-1, k}$ and $e_{k, k+1}$ of the $(n+1)$-gon. That is, consider the maximal sequence of edges having the form $e_{j_{r}, k}, e_{j_{r-1}, k}, \ldots, e_{j_{1}, k}, e_{k, l_{s}}, e_{k, l_{s-1}}, \ldots, e_{k, l_{1}}$ for $j_{1}<j_{2}<\cdots<j_{r}<k<l_{1}<l_{2}<\cdots<l_{s}$. When there exists some $w$ such that $v \prec w$ with $\lambda(v, w)=k$, this implies $r \geq 2$ and $s \geq 1$. Notice that for a cover relation $v \prec w$ to have $\lambda(v, w)=k$, it must be replacing the edge $e_{j_{1}, k}$ in $v$ by an edge $e_{j_{2}, l_{s}}$ to obtain $w$. In particular, this means there is at most one cover relation $v \prec w$ colored $k$.

Lemma 5.7. Suppose $v$ is covered by distinct elements $u_{i}(v)$ and $u_{j}(v)$ for $i<j$. If $u_{i} u_{j}(v)=$ $u_{j} u_{i}(v)$, then the interval $\left[v, u_{i}(v) \vee u_{j}(v)\right]$ has exactly 4 elements. If $u_{i} u_{j}(v) \neq u_{j} u_{i}(v)$, then $u_{i} u_{j}(v)=u_{j} u_{j} u_{i}(v)$, and the interval $\left[v, u_{i}(v) \vee u_{j}(v)\right]$ has exactly 5 elements, namely the elements $v, u_{i}(v), u_{j}(v), u_{i} u_{j}(v)$ and $u_{j} u_{i}(v)$. In either case, $u_{i}(v) \vee u_{j}(v)=u_{i} u_{j}(v)$.

Proof. In the case where $v \prec u_{i}(v)$ replaces an edge across one quadrilateral of $v$ while $v \prec u_{j}(v)$ replaces an edge across another quadrilateral of $v$ whose interior is completely disjoint from the interior of the first quadrilateral, these two edge replacement operations commute, yielding $u_{i} u_{j}(v)=u_{j} u_{i}(v)$; by construction, the interval from $v$ to $u_{i} u_{j}(v)$ will then have exactly 4 elements. To see that $u_{i} u_{j}(v)=u_{i}(v) \vee u_{j}(v)$ in this case, we use that the Tamari lattice is a lattice and that there is not room for a strictly lower upper bound for $u_{i}(v)$ and $u_{j}(v)$ by virtue of the definition of cover relation.

Suppose on the other hand that the pair of quadrilaterals that are triangulated by the two edges to be flipped from their positions in $v$ by the two cover relations $v \prec u_{i}(v)$ and $v \prec u_{j}(v)$ have interiors that are not disjoint. Then these triangulated quadrilaterals must share a triangle. This forces the union of the two triangulated quadrilaterals to comprise a triangulated pentagon appearing within the triangulation $v$. In order to have cover relations $v \prec u_{i}(v)$ and $v \prec u_{j}(v)$ both proceeding upward from $v$, one may check directly that the triangulated pentagon within $v$ must have 5 vertices labeled $a, b, i, j, k$ for $a<b<i<j<k$ with edges $e_{a, i}$ and $e_{a, j}$ that can be flipped by applying the operators $u_{i}$ and $u_{j}$, respectively, to give the new edges $e_{b, j}$ and $e_{i, k}$, respectively. One may likewise check directly that $\left[v, u_{i} u_{j}(v)\right]$ has exactly the desired 5 elements. By virtue of the incomparability of $u_{i}(v)$ and $u_{j}(v)$ together with the definition of cover relation, there cannot be any $z$ satisfying the three conditions $u_{i}(v) \leq z$ and $u_{j}(v) \leq z$ and $z<u_{i} u_{j}(v)$. Therefore, $u_{i} u_{j}(v)$ is a least upper bound for $u_{i}(v)$ and $u_{j}(v)$. Since the Tamari lattice is well known to be a lattice, this must be the unique least upper bound $u_{i}(v) \vee u_{j}(v)$. 


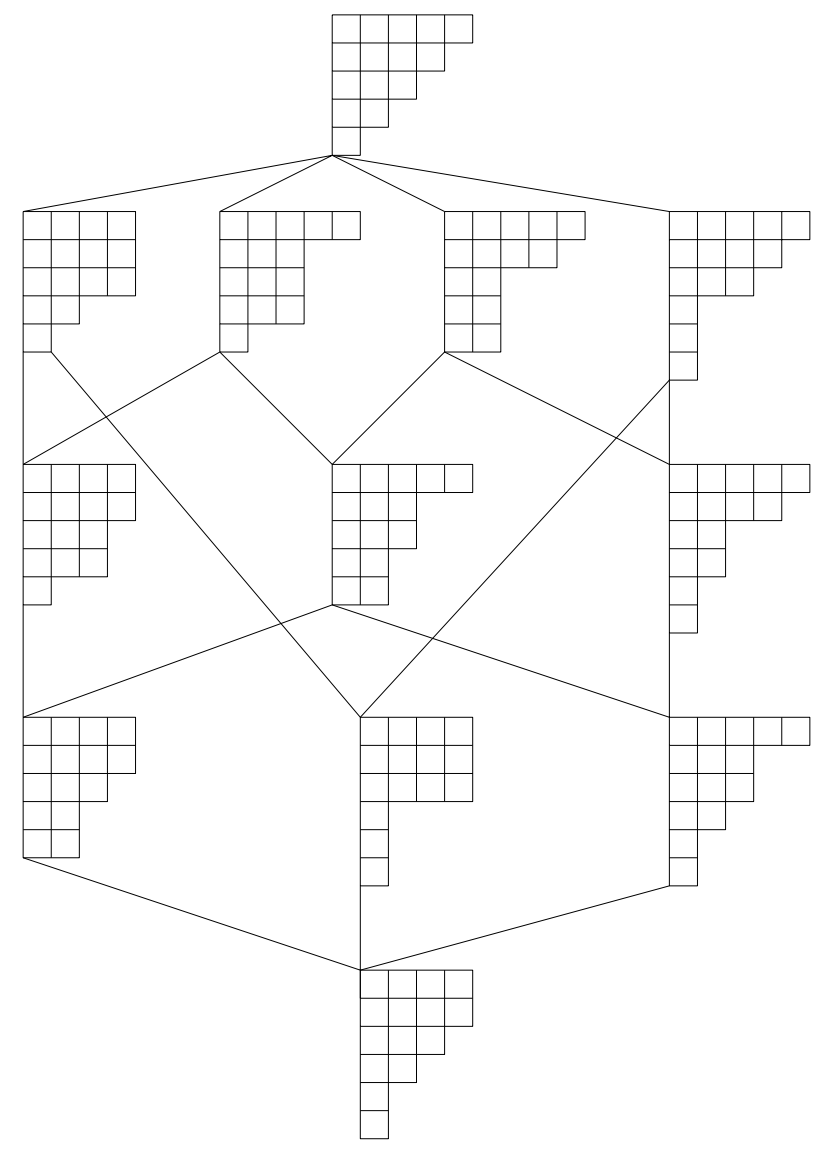

Figure 3. The interval between partitions $(4,4,3,2,1,1)$ and $(5,4,3,2,1)$ in the dominance order. This interval, as well as its dual, has no $S B$-labeling.

Example 5.8. Neither the dominance order on the partitions of an integer $n$ nor its dual poset admits an $S B$-labeling in general. This can be seen by considering the interval downward from the partition $(5,4,3,2,1)$ to the meet of the 4 elements that are covered by $(5,4,3,2,1)$, namely the interval shown in Figure 3 , we leave it as an exercise for the reader to check that neither the poset shown in Figure 3 nor its dual poset admits an $S B$-labeling, implying the same for dominance order for $n=15$. Note that the dominance order is an $I$-lattice, a notion introduced by Greene in [15] for proving that certain lattices take only Möbius function values $0,1,-1$. This example shows that the notions of $I$-lattices and $S B$-lattices are distinct.

Recall that the dominance order was proven to be non-pure shellable with each open interval homotopy equivalent to a ball or a sphere in 88. The Möbius function was determined prior to the development of the notion of non-pure shellability in [9], [10] and [15].

\section{ACKNOWLEDGMENTS}

The authors are grateful to Georgia Benkart, Stephanie van Willigenburg, Monica Vazirani, and the Banff International Research Station (BIRS) for conducting an inspiring workshop entitled Algebraic Combinatorixx in May 2011 for female researchers in algebraic combinatorics with the goal of helping establish new and fruitful collaborations. This project grew 
out of discussions that began at that workshop. The authors also thank Louis Billera, Anders Björner, Curtis Greene, Thomas McConville, Peter McNamara, Vic Reiner, Hugh Thomas, and the very helpful anonymous referees for helpful discussions, references, and suggestions.

\section{REFERENCES}

[1] A. Björner, Shellable and Cohen-Macaulay partially ordered sets, Trans. Amer. Math. Soc. 260 (1980), no. $1,159-183$.

[2] A. Björner, Homotopy type of posets and lattice complementation, J. Combin. Theory, Ser. A 30 (1981), $90-100$.

[3] A. Björner, Topological methods in combinatorics, Handbook of Combinatorics, Vol. 2, 1819-1872, Elsevier, Amsterdam, 1995.

[4] A. Björner, Orderings of Coxeter groups. Combinatorics and algebra (Boulder, Colo., 1983), 175-195, Contemp. Math., 34, Amer. Math. Soc., Providence, RI, 1984.

[5] A. Björner and F. Brenti, Combinatorics of Coxeter groups, Graduate Texts in Mathematics, 231, Springer, New York, 2005. xiv +363 pp.

[6] A. Björner, P. Edelman, and G. Ziegler, Hyperplane arrangements with a lattice of regions, Disc. and Comput. Geom. 5 (1990), 263-288.

[7] A. Björner and M. Wachs, On lexicographically shellable posets, Trans. Amer. Math. Soc., 277 (1983), no $1,323-341$.

[8] A. Björner and M. Wachs, Shellable and nonpure complexes and posets, II, Trans. Amer. Math. Soc. 349 (1997), 3945-3975.

[9] K. Bogart,The Möbius function of the domination lattice, unpublished manuscript, 1972.

[10] T. Brylawski, The lattice of integer partitions, Discrete Math 6 (1973), 201-219.

[11] P. Edelman, A partial order on the regions of $\mathbb{R}^{n}$ dissected by hyperplanes, Trans. Amer. Math. Soc. 283, no. 2 (1984), 617-631.

[12] P. Edelman and V. Reiner, The higher Stasheff-Tamari posets, Mathematika 43 (1996), 127-154.

[13] P. Edelman and J. Walker, The homotopy type of hyperplane posets, Proc. Amer. Math. Soc. 94 (1985), 221-225.

[14] J. Folkman, The homology groups of a lattice, J. Math. Mech. 15 (1966), 631-636.

[15] C. Greene, A class of lattices with Möbius function $\pm 1,0$, Europ. J. Combinatorics (1988), 9, 225-240.

[16] P. Hersh and C. Lenart, From the weak Bruhat order to crystal posets, arXiv:1510.05636 to appear in Math. Zeitscrift.

[17] S. Huang and D. Tamari Problems of associativity: A simple proof for the lattice property of systems ordered by a semi-associative law, J. Combin. Theory Ser. A 13 (1972), 7-13.

[18] J. Humphreys, Reflection Groups and Coxeter Groups, Cambridge Studies in Advanced Mathematics, 29, Cambridge University Press, 1990.

[19] D.E. Knuth, Computer Musings: The associative law, or The anatomy of rotations in binary trees, Distinguished Lecture Series VII (Stanford, CA: University Video Communications, 1993), 68 minutes (videotape).

[20] J.-L. Loday, Realization of the Stasheff polytope, Arch. Math. 83 (2004),267-278.

[21] H. Mühle, $S B$-labelings, distributivity, and Bruhat order on sortable elements, Electronic J. Combinatorics, $22(2), 2015$.

[22] J. M. Pallo, Enumerating, ranking and unranking binary trees, Computer J. 29 (1986), 171-175.

[23] J.M. Pallo, An algorithm to compute the Möbius function of the rotation lattice of binary trees, Informatique Theor. et. Applic./Theor. Informatics and Applic. 27 (1993), 341-348.

[24] G.-C. Rota, On the foundations of combinatorial theory I: Theory of Möbius functions, Z. Wahrscheinlichkeitstheorie u. Verw. Gebiete 2 (1964), 340-368.

[25] R. Stanley, Supersolvable lattices, Algebra Universalis 2 (1972), 197-217.

[26] R. Stanley, Enumerative Combinatorics, vol. 1, Second Edition, Cambridge Studies in Advanced Mathematics, no. 49, Cambridge University Press, Cambridge, 2012. 
[27] J. Stasheff, Homotopy associativity of H-spaces. I., II., Trans. Amer. Math. Soc. 108 (1963), 275-292, 293-312.

[28] J. Stembridge, A local characterization of simply laced crystals, Trans. Amer. Math. Soc. 355 (2003), 4807-4823.

Department of Mathematics, North Carolina State University, Raleigh, NC 27695

E-mail address: plhersh@ncsu.edu

Department of Mathematics, Cornell University, Ithaca, NY 14853

E-mail address: karola@math.cornell.edu 